\title{
The Arabidopsis CURVY1 (CVY1) gene encoding a novel receptor-like protein kinase regulates cell morphogenesis, flowering time and seed production
}

Emma W Gachomo ${ }^{1,2+}$, Lyla Jno Baptiste ${ }^{1+}$, Timnit Kefela', William M Saidel ${ }^{1,2}$ and Simeon O Kotchoni ${ }^{1,2^{*}}$

\begin{abstract}
Background: A molecular-level understanding of the loss of CURVY1 (CVY1) gene expression (which encodes a member of the receptor-like protein kinase family) was investigated to gain insights into the mechanisms controlling cell morphogenesis and development in Arabidopsis thaliana.

Results: Using a reverse genetic and cell biology approaches, we demonstrate that CVY1 is a new DISTORTED gene with similar phenotypic characterization to previously characterized ARP2/3 distorted mutants. Compared to the wild type, cvy 1 mutant displayed a strong distorted trichome and altered pavement cell phenotypes. In addition, cry 1 null-mutant flowers earlier, grows faster and produces more siliques than WT and the arp2/3 mutants. The CVY1 gene is ubiquitously expressed in all tissues and seems to negatively regulate growth and yield in higher plants.

Conclusions: Our results suggest that CURVY1 gene participates in several biochemical pathways in Arabidopsis thaliana including (i) cell morphogenesis regulation through actin cytoskeleton functional networks, (ii) the transition of vegetative to the reproductive stage and (iii) the production of seeds.
\end{abstract}

Keywords: CURVY1, Cell morphogenesis, Arabidopsis thaliana, Distorted trichome, T-DNA knockout mutant, Actin bundle, Protein kinase, Seed production

\section{Background}

In plants, cell shape patterning and growth are regulated by multiple genes that are mediated by actin and microtubule cytoskeleton-dependent trafficking pathways [1-3]. The combined activities of the cytoskeleton, endomembrane, and cell wall biosynthetic systems organize the cytoplasm and define the architectural cell patterning [1-3]. Genetic screens have identified a class of mutants known as DISTORTED mutants because of their significant actin-related cytoskeletal growth-associated phenotypic defects and overall distorted cell shape patterning

\footnotetext{
* Correspondence: simeon.kotchoni@rutgers.edu

${ }^{\dagger}$ Equal contributors

'Department of Biology, Rutgers University, 315 Penn St, Camden, NJ 08102, USA

${ }^{2}$ Center for Computational and Integrative Biology, 315 Penn St, Camden, NJ 08102, USA
}

and abnormal polarized growth (trichome, epidermis, cellcell communication) [2,4-6].

Genetic analysis reveals that gene that function in signal transduction cascades controlling local actin polymerization through the ARP2/3 complex [7-10] and the SCAR/WAVE complex [5,11-18] regulate cell patterning/morphogenesis in plants. Most of this knowledge comes from studies of differently distorted trichome mutants generally characterized by irregular cell expansion and polarized growth $[2,4,19,20]$.

In order to decipher the genetic basis of plant cell shape patterning and growth, we employed, in this study, a reverse genetic approach by screening the loss of gene expressions in Arabidopsis T-DNA knockout mutants to gain insights into the mechanisms controlling cell morphogenesis in plants. DISTORTED mutants are known to display a dramatic cell shape alteration in comparison 
to wild type plants. The overall cell (trichome, pavement cell, root system) morphology of DISTORTED mutants has been well studied [21]. The DISTORTED genes have been reported to function in signal transduction cascades that control actin cytoskeleton assembly through WAVE/SCAR2-ARP2/3 pathway [2,3,20,21].

In this manuscript, we describe a new DISTORTED gene termed CURVY1 (CVY1) that encodes a member of the receptor-like kinase (RLK) superfamily. Protein kinases are generally involved in perception of general elicitors initiating signal transduction cascades regulated by protein phosphorylation [22] to activate downstream responses that include the production of reactive oxygen species, ethylene biosynthesis, activation of a MAPK cascade, activation of abiotic or defense gene expression and other biological processes [23-26]. In addition, RLKs have also been recently related to the regulation of unidimensional cell growth, response to nitrate, and transferase activities in eukaryotes [22]. several protein kinases and their biological phosphorylation processes are still largely uncharacterized in Arabidopsis thaliana. Among the protein kinase genes, the CURVY1 (CVY1) gene appears to have a unique function related to cell morphogenesis, as cvy1 mutant displays phenotypes similar to distorted SCAR/WAVE and ARP2/3 mutant cell morphologies $[2,4,16,27]$. Using a reverse genetic approach, we examined and characterized a SALK_T-DNA knockout curvy1 mutant (cvy1) with respect to cell morphogenesis and growth phenotypes. Knockout mutation in CVY1 caused severe trichome growth defects with relatively mild effects on overall shoot development, demonstrating that $C V Y 1$ functions in polarized cell growth and cell shape patterning. In addition, the work demonstrates that CURVY1 represents a novel receptorlike kinase that regulates trichome, pavement cell morphogenesis and cell wall biogenesis among other interesting phenotypic features and might function in signal transduction cascades that control local actin assembling through the SCAR2/WAVE-ARP2/3 pathway.

\section{Results and discussion}

Genetic and phenotypic characterization of curvy 1 mutant To investigate the role of CURVY1 in regulating cell morphogenesis in plants, we initiated a reverse genetic analysis of the gene using the Salk collection of Arabidopsis TDNA knockout lines of our in-house Arabidopsis seed stock library. CURVY1 is here shown to be important not only for polarized cell growth and trichome morphology but also other biological processes including flowering time and seed production. Our data reveals that mutations in CURVY1 gene results in strong-distorted trichomes that are similar to the SCAR/WAVE and ARP2/3 mutant phenotypes [2,5,7-18]. To our knowledge, this is the first time that CURVY1 has been shown to control cell morphology/patterning (Figure 1). In addition, we investigated the role of CURVY1 in other biological processes. We employed a reverse genetic approach using the Arabidopsis T-DNA SALK lines mediating loss of function of CURVY1 gene to examine curvy1-knockout phenotypes. The SALK_018797 (curvy1) line harboring a T-DNA insertion in the only exon of CURVY1 gene map (Figure 1A) was selected and confirmed as null mutant with loss of CVY1 function. We confirmed the location of the T-DNA using the T-DNA-specific oligonucleotide primer LB1 and the CVY1-specific primer (Table 1) and examined the CVY1 mRNA transcript levels in wild type and cvy1 mutant using RT-PCR. As shown in (Figure 1B), the T-DNA insertion caused a knockout of the CVY1 gene in cvy1 mutant background. The mutation caused significant distortion of trichomes (Figure 1C, D, Table 2) and altered pavement cell morphology (Figure 1E, F, Table 3) compared to wild type. The $c v y 1$ cell patterning (trichomes,

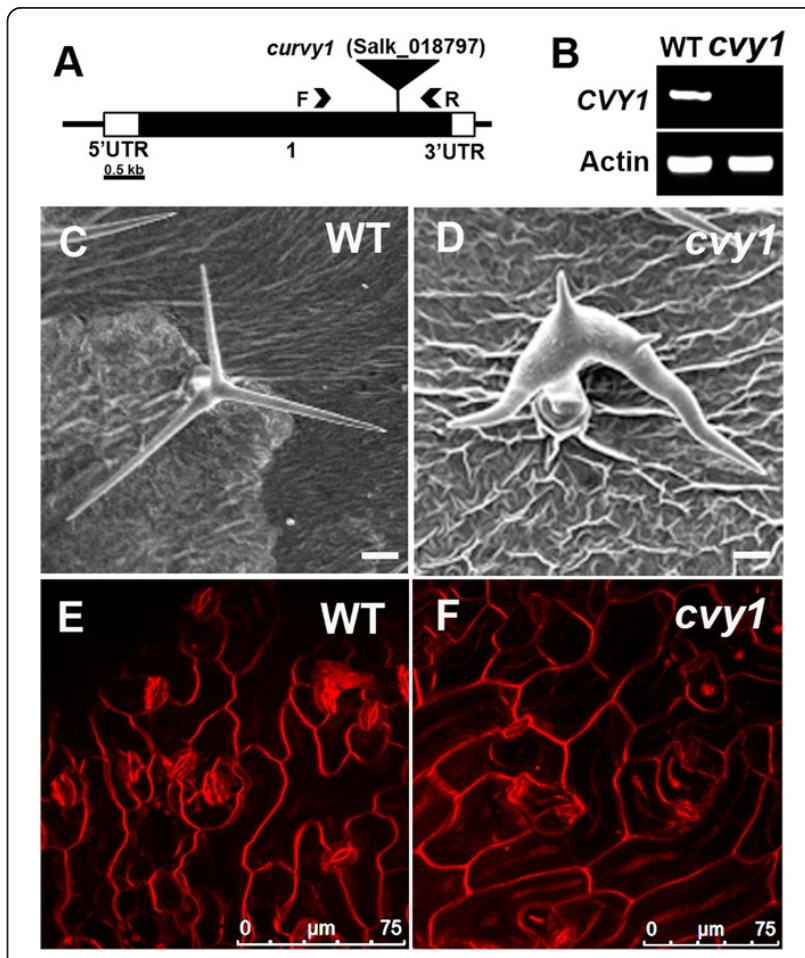

Figure 1 Physical map of CVY1 gene knockout and phenotypic characterization of cvy1 mutant. (A) The CVY1 gene with the positions of the exon (numbered black rectangle) of the gene represented. The $5^{\prime}$ and $3^{\prime}$ untranslated regions are depicted in white rectangles. The location of the Salk T-DNA insertion is shown using an inverted black triangle. The names and locations of primers used for RT-PCR analysis are also indicated. Bar $=0.5 \mathrm{~kb}$. (B) The T-DNA insertion causes a knockout expression of the gene. The quality of the RNA and the loading control was assayed by monitoring ACTIN gene expression. (C and D) SEM images of upper developing leaves, showing a mature trichome with three branches in wild type (C) and strong distorted trichome in cry 1 (D) plants. (E and F) Confocal images of pavement cell shape pattern of 12 days old WT (E) and cry 7 (F) using lipophilic dye, FM464. Bars $=50 \mu \mathrm{m}$ (C, D). 
Table 1 Sequences of oligonucleotide primers used in this study

\begin{tabular}{lll}
\hline Name & Primer sequence & Description \\
\hline CVY1-F1 & 5'TGCGATGGAGACTGTTTCTCGTGT3' & For RT-PCR \\
CVY1-R1 & 5'ATCAGAGTTAACCTCGTGGCGGT3' & For RT-PCR \\
TDNA-LB & 5'CCGTCTCACTGGTGAAAAGAA3' & For TDNA insertion \\
CRV1-F2 & 5'ATCATCCCGGGTATCTTCTCCGAA & For complementation \\
& TATAGACT3' & test (Smal site italicized) \\
CVY1-R2 & 5'CAATTGCCCGGGATATATAATTTA & For complementation \\
& AGCTTCTTTGT' & test (Smal site italicized) \\
Act2-F & 5'GCGGATCCATGGCTGAGGCTGAT & For RT-PCR \\
& GATATTCAACC3' & \\
Act2-R & 5'CGTCTAGACCATGGAACATTTCTG & For RT-PCR \\
& TGAACGATTCC3' & \\
\hline
\end{tabular}

epidermal cells) is not obviously different from previously characterized arp2/3 (arpc2, arpc4) distorted mutants (Tables 2 and 3). The tissue specific expression pattern of CVY1 (Additional file 1: Figure S1) is consistent with Genevestigator microarray data [28]. The CURVY1 gene is ubiquitously expressed in all tested tissues, but particularly high in polarized cells/tissues such as the trichome, root, root tip, and hypocotyls (Additional file 1: Figure S1), suggesting its importance in plant cell morphogenesis and polarized cell growth.

\section{CURVY1 controls cell morphogenesis in plants}

We confirmed that cvy1 morphological phenotype was indeed caused by the described T-DNA insertion by constitutively overexpressing CVY1 gene in cvy1 mutant background. This complementation functionality test was performed by using Agrobacterium tumefaciens mediated transformation to introduce the 35-promoter-CVY1 transgene into $c v y 1$ plants [29]. As expected, overexpression of $C V Y 1$ in $c v y 1$ mutant background was sufficient to rescue the $c v y 1$ phenotype (Figure $2 \mathrm{~A}, \mathrm{~B}$ ), demonstrating that CVY1 gene knockout is indeed responsible for the phenotypic characterization in cvy1 mutant phenotype, and thus providing further confirmation of the correct

Table 2 Comparative quantitative phenotypic analysis of cvy 1 trichomes to well characterized arp2/3 trichome mutants

\begin{tabular}{lllll}
\hline Trichome & WT & curvy1 & arpc2 (dis2) & arpc4 \\
\hline Branch 1 $(\mu \mathrm{m})$ & $286 \pm 31$ & $82 \pm 27$ & $87 \pm 31$ & $78 \pm 26$ \\
& $(n=16) \mathrm{a}$ & $(\mathrm{n}=10) \mathrm{d}$ & $(\mathrm{n}=14) \mathrm{d}$ & $(\mathrm{n}=12) \mathrm{d}$ \\
Branch 2 $(\mu \mathrm{m})$ & $256 \pm 50$ & $30 \pm 10$ & $29 \pm 8$ & $28 \pm 12$ \\
& $(\mathrm{n}=16) \mathrm{b}$ & $(\mathrm{n}=10) \mathrm{e}$ & $(\mathrm{n}=14) \mathrm{e}$ & $(\mathrm{n}=12) \mathrm{e}$ \\
Branch 3 $(\mu \mathrm{m})$ & $196 \pm 46$ & $22 \pm 12$ & $18 \pm 7$ & $20 \pm 8$ \\
& $(n=16) \mathrm{c}$ & $(\mathrm{n}=10) \mathrm{f}$ & $(\mathrm{n}=14) \mathrm{f}$ & $(\mathrm{n}=12) \mathrm{f}$
\end{tabular}

The numbers in the parentheses indicate the number of samples analyzed. Mean values with different letters are significantly different from each other, and mean values with the same letter in the group are not significantly different $(P<0.05)$.
Table 3 Comparative quantitative analysis of cvy1 pavement cell shape phenotype to well characterized arp2/3 pavement cells

\begin{tabular}{lllll}
\hline Pavement cell & WT & curvy1 & arpc2 (dis2) & arpc4 \\
\hline Size $\left(\mu m^{2}\right)$ & $\begin{array}{l}2.10 \pm 0.6 \\
(n=25) a\end{array}$ & $\begin{array}{l}1.56 \pm 0.3 \\
(n=24) d\end{array}$ & $\begin{array}{l}1.70 \pm 0.61 \\
(n=20) d\end{array}$ & $\begin{array}{l}1.62 \pm 0.31 \\
(n=28) d\end{array}$ \\
Circularity* $^{0.25 \pm 0.06}$ & $\begin{array}{l}0.38 \pm 0.05 \\
(n=25) a\end{array}$ & $\begin{array}{l}0.34 \pm 0.06 \\
(n=24) d\end{array}$ & $\begin{array}{l}0.30 \pm 0.03 \\
(n=20) d\end{array}$ \\
\hline
\end{tabular}

The numbers in the parentheses indicate the number of samples analyzed. Mean values with different letters are significantly different from each other, and mean values with the same letter in the group are not significantly different $(P<0.05)$. ${ }^{*}$ Circularity describes the cell shape complexity.

genetic characterization of CURVY1 as a new "DISTORTED" gene. The T-DNA (SALK_018797) causing knockout mutation in CVY1 (At2g39360) is also present in MIR156A (At2g25095) gene that targets SPL3. However, we ruled out the possibility of $c v y 1$ mutant phenotypes being caused by a plausible insertion on MIR156A gene because homozygous mir156A mutant does not have distorted trichome phenotype and the overall phenotypic complementation tests (trichome phenotype, flowering time, seed production and hypocotyl gravitropism) excluded the implication of MIR156A mutation in the observed/described curvy1 phenotypes (Table 4).

Trichome branch length assay and pavement cell phenotypes are generally the most sensitive assays to describe phenotypic similarity among different distorted mutants $[2,20]$. The trichome phenotypes (Table 2) of curvy1 mutants were indistinguishable from the well characterized $A R P 2 / 3$ distorted mutants (ARPC2 and ARPC4). In addition, $c v y 1$ shape complexity of pavement-cells was significantly reduced compared to WT (Figure 1E, F), but was also indistinguishable from arpc2 and arpc4 pavement cells (Figure 3A-D, Table 3), suggesting a conserved cell shape regulatory relationship between CURVY1 and $A R P 2 / 3$ in plants. The data suggests that CURVY1 belongs to the "distorted group" of genes. ARP2/3 gene mutations are associated with actin cytoskeleton defects [2], suggesting that

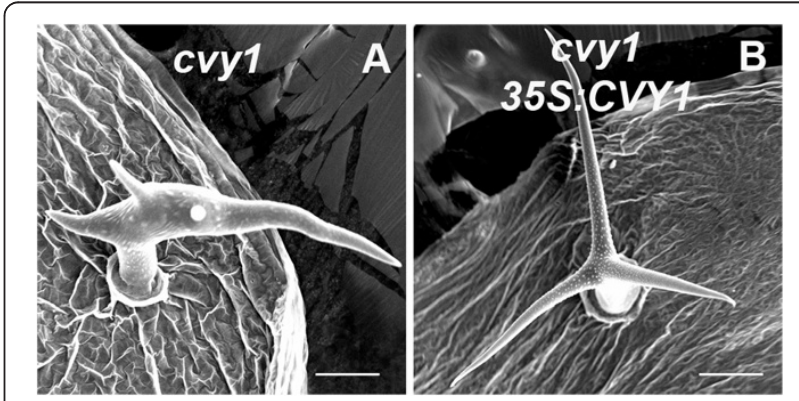

Figure 2 Overexpression of CVY1 gene rescues the cvy 1 trichome phenotype in a complementation test. A) Distorted trichome phenotype of cry 1 mutant. B) The distorted trichome phenotype in cvy 1 mutant is perfectly rescued by $35 \mathrm{~S}: \mathrm{CVY} 1$ gene expression. 
Table 4 Overexpression of CVY1 gene rescues the overall cvy1 phenotypes in complementation tests

\begin{tabular}{llll}
\hline Phenotypes & WT & curvy1 & $\begin{array}{l}\text { cvy1_35S: } \\
\text { CVY1 }\end{array}$ \\
\hline $\begin{array}{l}\text { Flowering time (in number } \\
\text { of rosette leaves) }\end{array}$ & $\begin{array}{l}14.0 \pm 1.5 \\
(\mathrm{n}=22) \mathrm{a}\end{array}$ & $\begin{array}{l}10.0 \pm 1.1 \\
(\mathrm{n}=28) \mathrm{b}\end{array}$ & $\begin{array}{l}15.5 \pm 2.0 \\
(\mathrm{n}=12) \mathrm{a}\end{array}$ \\
$\begin{array}{l}\text { Number of siliques/seed } \\
\text { production at 31 days }\end{array}$ & $\begin{array}{l}12.5 \pm 2.0 \\
(\mathrm{n}=22) \mathrm{a}\end{array}$ & $\begin{array}{l}45.0 \pm 5.0 \\
(\mathrm{n}=28) \mathrm{b}\end{array}$ & $\begin{array}{l}14.0 \pm 4.0 \\
(\mathrm{n}=12) \mathrm{a}\end{array}$ \\
$\begin{array}{l}\text { Dark grown phenotype } \\
\text { GG }(\mathrm{n}=22)\end{array}$ & LGG $(\mathrm{n}=28)$ & $\mathrm{GG}(\mathrm{n}=12)$ \\
\hline
\end{tabular}

Flowering time, siliques/seed production and dark phenotypes of cvy1 mutant were rescued by $35 \mathrm{~S}: \mathrm{CVY} 1$ gene expression in a complementation test.

Numbers in the parentheses indicate the number of samples analyzed. Mean values with different letters are significantly different from each other, and mean values with the same letter in the group are not significantly different $(P<0.05)$. GG = Grow against gravity; LGG = Loss of growth against gravity.
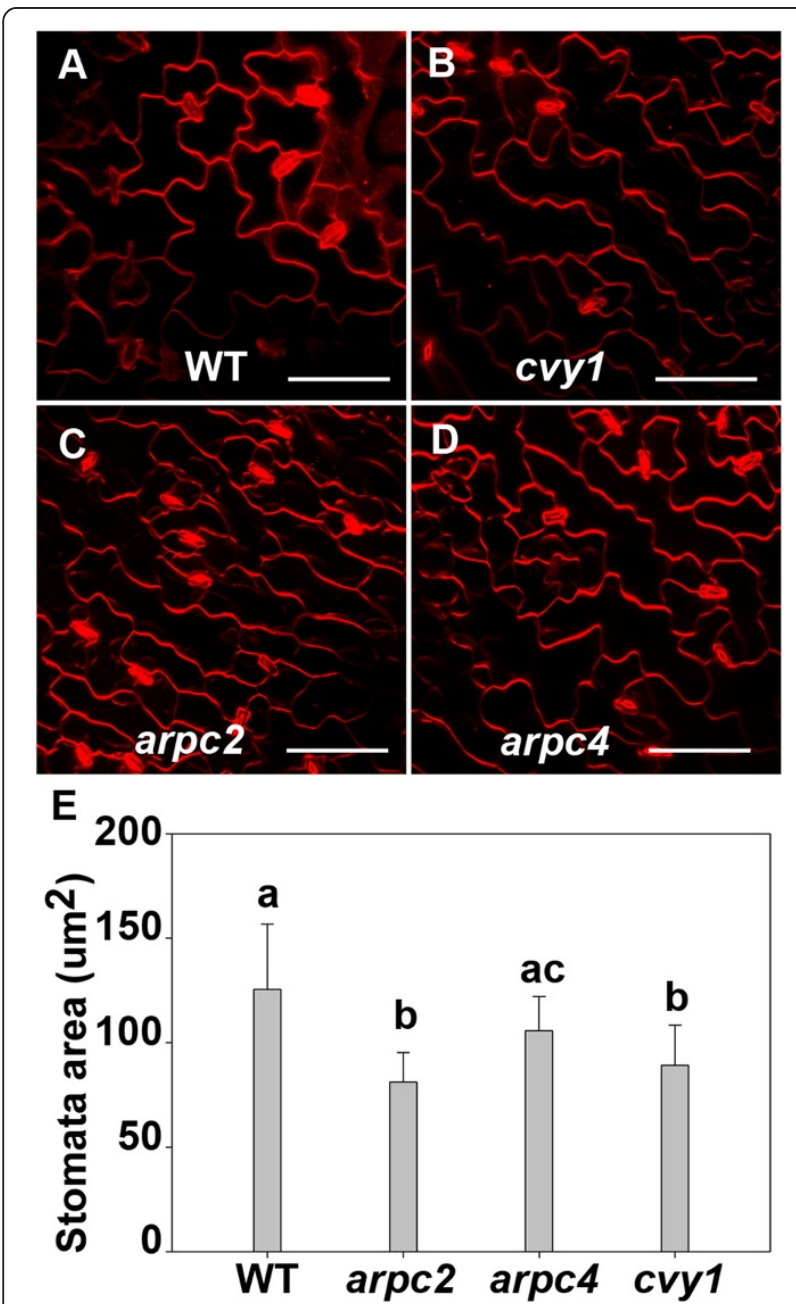

Figure 3 curvy 1 cell shape phenotype is indistinguishable from arp $2 / 3$ cell shape mutants. (A-D) Wide-field fluorescence images of fields of cotyledon epidermal pavement cells of wild-type (A), cvy1 (B), $\operatorname{arpc2}$ (C) and arpc4 (D). (E) Wild type-stomata are bigger than the mutant-stomata. Stomata mean values with different letters are significantly different from each other, and mean values with the same letter in the group are not significantly different $(P<0.05)$. Bars $=50 \mu \mathrm{m}$
CURVY1 might regulate cell morphogenesis through signal transduction cascades that control local actin assembly through the ARP2/3 complex or the SCAR/WAVE complex $[2,20]$. In addition, we scored the stomata surface areas and found WT-stomata overall to be bigger than the stomata of mutants (Figure 3E). We analyzed the growth of wild type, curvy1 mutants and the well characterized $\operatorname{arp2/3}(\operatorname{arpc} 2, \operatorname{arpc} 4)$ mutants under latrunculin B (LatB), an actin filament depolymerization drug [30]. The wild type ( $\mathrm{n}=22$ seedlings), curvy1 $(\mathrm{n}=28$ seedlings) and arp2/ 3 ( $\mathrm{n}=12$ seedlings) were affected by LatB $(5 \mathrm{nM})$ treatment. However, we observed a significantly stronger effect of LatB on curvy1 mutants that was indistinguishable from the effect of LatB on arp2/3 (arpc2 and arpc4) mutants (Table 5), supporting the notion that CURVY1 might regulate cell morphogenesis through actin cytoskeleton networks $[2,30]$. To further make the link between CURVY1 and the actin cytoskeleton, we tested the sensitivity of $c v y 1$ rescue lines to the actin depolymerization drug LatB. As expected, the effect of LatB on $c v y 1$ rescue lines ( $\mathrm{n}=20$ seedlings) were similar to that of the wild type $(n=22$ seedlings). Overall, these data demonstrate that CURVY1 regulates cell morphogenesis through actin cytoskeleton functional network.

\section{CURVY1 encodes a member of the receptor-like kinase} (RLK) protein family

The RLKs are integral plasma membrane associated proteins with an extracellular domain that mainly binds to a carbohydrate, a transmembrane domain, and an intracellular Ser/Thr kinase domain [31]. Overall, plant RLKs have been reported to regulate various signaling pathways, including meristem function, brassinosteroid perception, floral abscission, ovule development and embryogenesis, plant defense, and plant morphology [32]. Previous studies showed that selected members of Arabidopsis CrRLK gene family including FERONIA (FER: At3g51550) [33-36], THESEUS1 (THE1: At5g54380) [37], HERCULES1 [35], ANXUR1 and ANXUR2 (ANX1 and ANX2) [38,39] regulate cell growth processes in different tissues under different development conditions.

Table 5 The effect of latrunculin B (LatB) on wild type arp2/3 and cvy1 seedlings

\begin{tabular}{lllll}
\hline Treatment & \multicolumn{4}{l}{ Root length $(\mathbf{m m})$} \\
\cline { 2 - 5 } & WT & curvy1 & arpc2 (dis2) & arpc4 \\
\hline Control & $15.5 \pm 0.5$ & $15.0 \pm 0.3$ & $10.0 \pm 0.6$ & $9.0 \pm 0.3$ \\
& $(n=22) \mathrm{a}$ & $(\mathrm{n}=28) \mathrm{a}$ & $(\mathrm{n}=12) \mathrm{b}$ & $(\mathrm{n}=12) \mathrm{b}$ \\
LatB (5 nM) & $7.0 \pm 0.05$ & $5.0 \pm 0.05$ & $4.5 \pm 0.06$ & $4.0 \pm 0.03$ \\
& $(\mathrm{n}=22) \mathrm{c}$ & $(\mathrm{n}=28) \mathrm{d}$ & $(\mathrm{n}=12) \mathrm{d}$ & $(\mathrm{n}=12) \mathrm{d}$ \\
\hline
\end{tabular}

The numbers in the parentheses indicate the number of samples analyzed. Mean values with different letters are significantly different from each other, and mean values with the same letter in the group are not significantly different $(P<0.05)$. The data was generated from vertical plate grown seedlings for 12 days under continuous illumination. 
Likewise, CURVY1 has been found to control plant cell morphology and overall growth including flowering time, cell polarity, and actin cytoskeleton network.

CURVY1 gene encodes a receptor-like kinase (RLK) that belongs to the Catharanthus roseus RLK (CrRLK)-like family $[40,41]$. RLKs represent a large diverse family of proteins with approximately 600 members in Arabidopsis thaliana [42]. However, the CrRLK-like family comprises a conserved extracellular carbohydrate-binding malectin-like domain [40] with 17 members in Arabidopsis (Figure 4) and 20 in rice [40]. As expected, CURVY1 displayed all protein features (malectin-like domain, serine/threonineprotein kinase active site, protein kinase catalytic domain) of well characterized CrRLK-like family (Figure 4A, [41]). Interestingly, all 17 Arabidopsis members of CrRLK gene family are structurally well conserved. They are exclusively made of a single exon flanked with a variable UTR length structure at both 3' and 5' ends. Nine out of the 17 Arabidopsis members of CrRLK1-like gene family are located on chromosome 5, three on chromosome 2 and 3 respectively and one on chromosome 1 and 4 respectively (Figure 4B). Phylogenetic analysis revealed four subclasses with CURVY1 belonging to the larger subclass composed of 10 members including the well characterized THESEUS1 (THE1: At5g54380) and HERCULES1 (HERK1: At3g46290) (Figure 4B). These four subclasses suggest a diversification of Arabidopsis CrRLK1-like proteins based on functional specifications (Figure 4B).

Actin bundles are disorganized in curvy 1 epidermal cells We examined the organization of the actin cytoskeleton in pavement cells of cvy1 mutant. The wild type $(\mathrm{n}=8)$ generates a significantly higher population of polarized actin bundles extending towards to the peripheral patterning of the pavement cells (Figure 5A). The $c v y 1$ pavement cells $(n=10)$ displayed the presence of high levels of presumably diffuse and loosely aligned actin monomers and filaments, but lacking in polarized actin bundles (Figure 5B). The actin cytoskeleton phenotype of cvy1

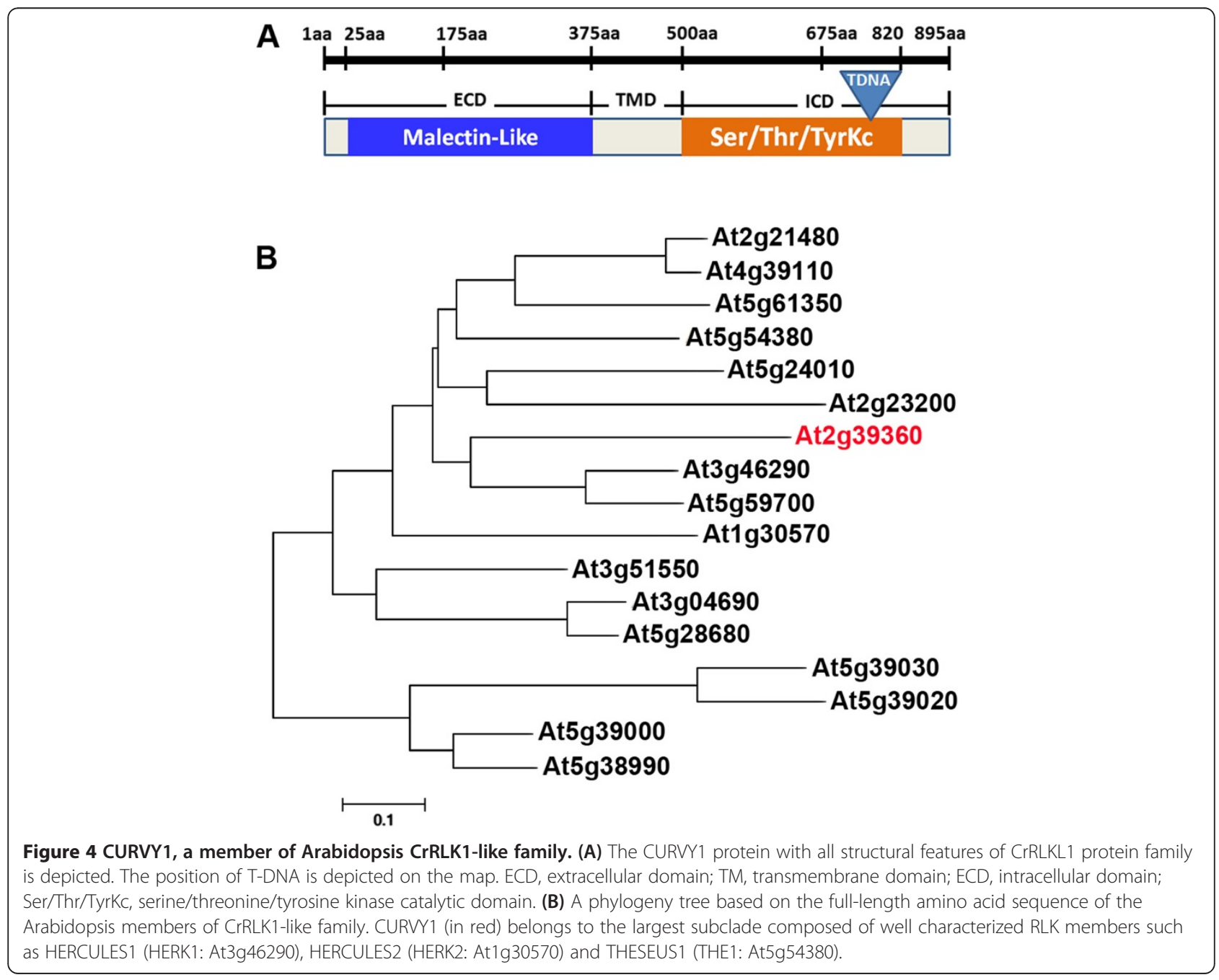




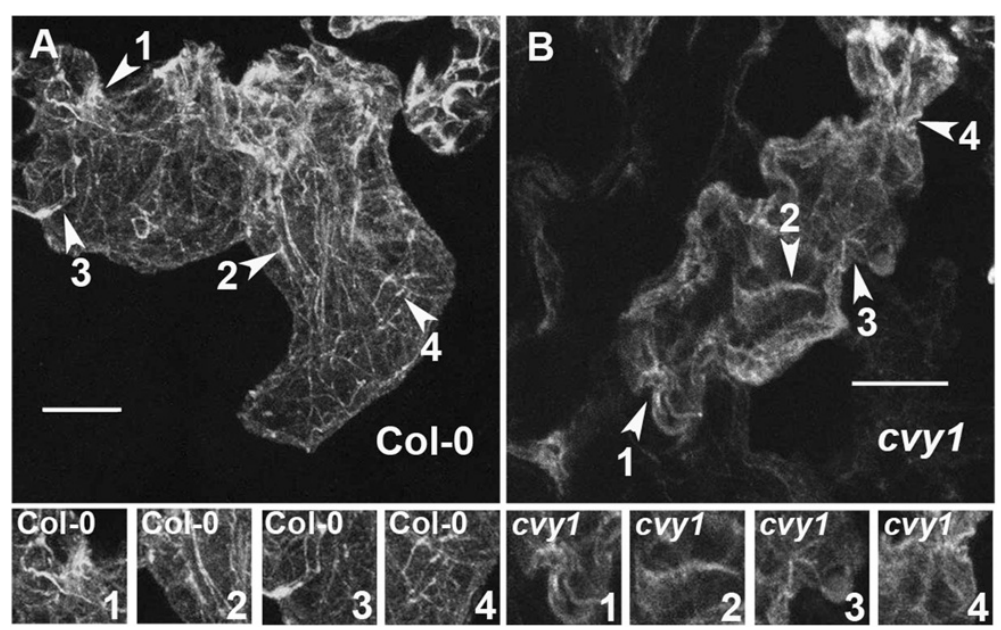

Figure 5 Knockout cvy1 null mutant displays reduced and disorganized actin bundles. (A and B) Actin organization in wild-type and curvy1 pavement cells was visualized using fluorescent phalloidin as previously described [2]. Depicted regions (arrow heads with numbers) of WT and curvyl pavement cells were magnified in the bottom panels to display the actin bundles in respective genotype backgrounds. Bars $=10 \mu \mathrm{m}$.

mutant is similar to what has been reported for arp $2 / 3$ mutants [2]. The actin cytoskeleton phenotype (Figure 5) suggests that the CURVY1 gene might function in a common WAVE/SCAR2-ARP2/3 pathway [2,3,6,20,30]. To further support the function of CURVY1 through actin cytoskeleton network, we used the Image analysis tool to quantify the number of actin bundles $(\mathrm{AB})$ in pavement cells after thresholding the stacked image to easily track/ count the actin bundles. Using a grid system (of $25 \mu \mathrm{sq}$ surface area as unit of the grid) covering the entire pavement cell (Additional file 2: Figure S2), we obtained a significantly $(\mathrm{P}<0.05)$ higher number of actin bundles in WT $(\mathrm{AB}=6.333 \pm 1732, \mathrm{n}=9$ samples $)$ compared to $c v y 1 \mathrm{mu}-$ tant $(\mathrm{AB}=2.333 \pm 1414, \mathrm{n}=9$ samples $)$ per surface unit of the grid (Additional file 2: Figure S2). Consistent with the diffused and loosely aligned actin cytoskeleton phenotype of curvy1 (Figure 5), the dark grown cvy1 mutant displayed a loss of gravity and polarized growth orientation compared to WT (Figure 6A, B). In addition, the etiolated phenotype was rescued by overexpressing CURVY1 gene in $c v y 1$ mutant plants (Figure 6C, Table 4), suggesting that CURVY1 regulates cell morphology and polarized growth through functional actin cytoskeleton network in Arabidopsis thaliana.

\section{CURVY1 controls other biological processes in plants}

Interestingly, we noticed an early flowering phenotype in cvy1 mutants $(\mathrm{n}=18)$ supported by a significantly ( $\mathrm{P}<0.05)$ reduced number of rosette leaves $(9 \pm 0.8)$ compared to the wild type $(13 \pm 0.5, \mathrm{n}=16)$ at bolting time. Unlike cvy1 mutants, arp $2 / 3$ mutants (arpc 2 rosette leaves $=18 \pm 1.3, \mathrm{n}=14$ and $\operatorname{arpc} 4$ rosette leaves $=$
$22 \pm 1.5, \mathrm{n}=12$, at bolting time) showed a significantly $(\mathrm{P}<0.05)$ delayed flowering phenotype compared to WT and cvy1 mutant (Figure 7A), suggesting that CURVY1 might regulate growth development through distinct signal transduction cascades to control transition from vegetative to reproductive stage. The homozygous cvy1 mutant $(n=9)$ displayed a faster growth rate and higher seed pod (siliques) production compared to the wild type $(\mathrm{n}=9)$ and $\operatorname{arp} 2 / 3$ mutants $(\mathrm{n}=9)$ (Figure $7 \mathrm{~B}-\mathrm{D})$, indicating that CURVY1 negatively regulates cell division and growth in meristemic regions as well as the overall production of seeds. Under similar growth conditions, cvy1 mutants produced about three-fold more seed pods (siliques: yield) compared to WT (Figure 7B) and 10-fold more siliques than $\operatorname{arpc} 2$ mutant (Figure 7C). Manipulating CURVY1 gene might be a promising target to improve crop yield in higher plants. To support this observation, we weighed all the seeds of each genotype at harvest time and found cvy1 seeds weighing two and half times more than those of WT and five times more than seeds of arpc 2 mutant. Our data reveals that mutations in $C V Y 1$ gene result in early flowering, senescence, and improved seed productivity. The mechanism by which CURVY1 regulates transition processes from vegetative to reproductive phase needs to be investigated in agronomically important crops.

\section{Conclusions}

In summary, we present in this work the identification of a new gene, CURVY1 that regulates growth, cell morphogenesis and seed production in Arabidopsis thaliana. This work presents evidence that CURVY1 belongs to 

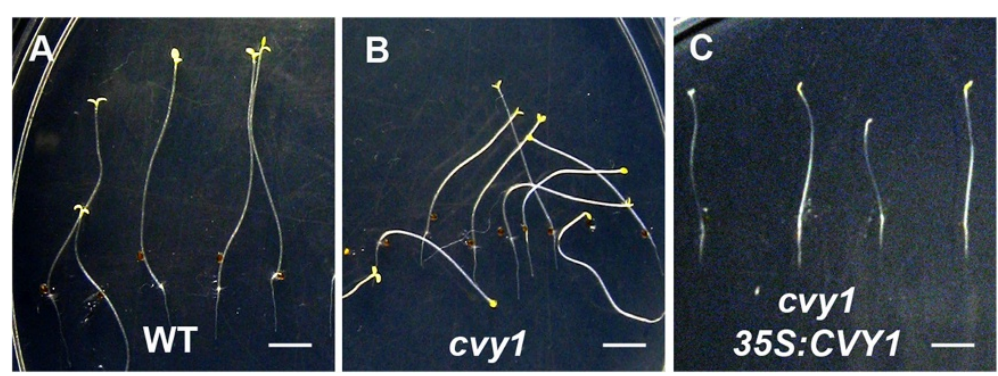

Figure 6 curvy 1 mutants displayed distinctly pronounced dark phenotypes. (A, B) wild type (A), cvy1 (B) and cVy1 35S:CVY1 rescue (C) seedlings grown on agar plates for 12 days after germination in the dark are here depicted. Under dark growth conditions, curvy 1 mutant (B) showed lack of vertical growth orientation compared to WT (A). The etiolated dark phenotype was perfectly rescued by overexpressing CVY 1 gene in the mutant background (C). Bars $=5 \mathrm{~mm}$.

the "distorted group" of genes. Homozygous cvy1 mutant displayed strong morphological phenotypes that are indistinguishable from the well-characterized DISTORTED trichome mutants [2]. The CURVY1 gene encoding a receptor-like protein kinase is ubiquitously expressed in all tissues tested. The distorted trichome phenotype in cvy1 mutant was rescued by expressing CURVY1 gene in the mutant background. Unlike the other DISTORTED mutants, mutation of CURVY1 gene promotes early flowering and seed production in Arabidopsis thaliana. Overall, CURVY1 represents a novel receptor-like kinase gene involved in regulating cell morphogenesis, including trichome and pavement cell shape patterning through local actin cytoskeleton assembling and additionally functions in signal transduction cascades that control flowering time and seed production in plants.

\section{Methods}

Plant strain, growth conditions and mutant characterization

Arabidopsis thaliana (ecotype Col-0) and cvyt1 knockout mutant (T-DNA SALK_018797) [from Arabidopsis Biological Research Center (ABRC)] were used throughout this work. Appropriate seeds were sown on Murashige and Skoog (1× MS) agar plates or soil and seedlings were allowed to grow under continuous illumination (120-150 $\mu \mathrm{Em}^{-2} \mathrm{~s}^{-1}$ ) at $24^{\circ} \mathrm{C}$. For $c v y 1$ mutant characterization, T-DNA insertion was PCR-confirmed using CVY1 gene specific primers (Table 1) and T-DNA left border primer Lb (Table 1). To analyze the expression of CVY1 gene in mutant backgrounds, total RNA was extracted from the homozygous T-DNA insertion mutants by TRIzol reagent (Molecular Research Center) and then reversed transcribed
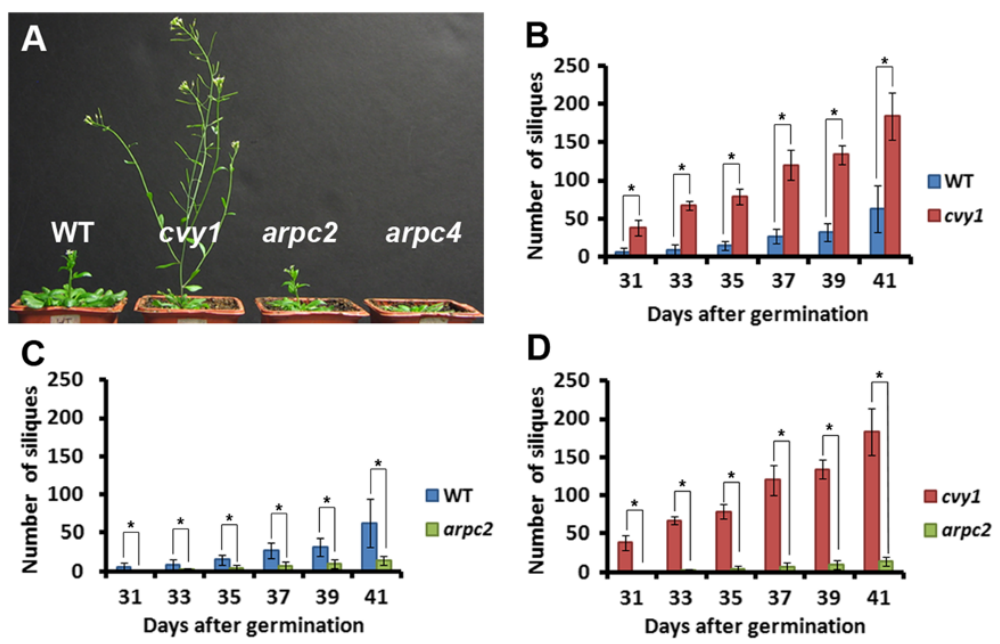

Figure 7 curvy 1 mutant flowers earlier and produce more seeds than WT and arp2/3 mutants. (A) Representative growth phenotype of the seedlings is depicted at 29 days after germination in soil. (B-D) Number of siliques produced at indicated days after germination. Comparative production of siliques between WT and cryl (B), WT and $\operatorname{arpc2}$ (C), cvy1 and $\operatorname{arpc2}$ (D) is depicted. No silique was produced by arpc4 mutant at 41 days after germination and no comparative data was done with arpc4 mutant. Means \pm STDEV of plants $(n=6)$ per genotype are shown. Significant differences in comparison analysis are indicated with asterisks: ${ }^{*} \mathrm{P}<0.05$. 
using qScript cDNA Supermix (Quanta BioSciences, Gaithersburg, MD, USA) as previously described [30]. Thereafter, the cDNA was used as template for PCR using CVY1 gene-specific primers (Table 1), running 30 amplification cycles (linear range of amplification) [30]. PCR fragments were separated on $1 \%$ agarose gels containing ethidium bromide. A cDNA fragment generated from ACTIN served as an internal control.

For complementation test, a RT-PCR amplification of $2600 \mathrm{bp}$ fragment containing the 5' and 3' untranslated regions as well as CVY1-encoding sequence (At2g39360) from WT cDNA (Table 1) was cloned into the SmaI site of the pROK2 vector [43] in front of CaMV 35S promoter-driven overexpression $[43,44]$ and stably transformed cvy1 mutant background by the floral dip method [29]. For tissue specific gene expression analysis, the cDNA from respective tissues was used to perform real-time qPCR of CVY1 gene expression. Real-time qPCR was performed on Eco real-time PCR system (Illumina, San Diego, CA, USA) using PerfeCTa SYBR green FastMix (Quanta BioScience, Gaithersburg, MD, USA). The relative $C V Y 1$ expression level was assessed using ACTIN gene as internal control (Table 1).

\section{Arabidopsis thaliana CrRLK1-like family: structural characterization and phylogenetic analysis}

Catharanthus roseus RLK (CrRLK) characteristics were used to retrieve the 17 members of Arabidopsis thaliana CrRLK1-like gene family according to Hematy and Hofte [31] and used to generate the phylogenetic tree according to Gachomo et al. [30]. CURVY1 (a member of CrRLK1-like family) protein functional domains were studied using different structure-functional motifs and/ or patterns databases such as Pfam v25.0 (pfam.sanger. ac.uk), Prosite (prosite.expasy.org/scanprosite) and Conserved Domain Database (CDD) v3.02, CDART (Conserved Domain Architecture Retrieval Tool) to reveal the kinase catalytic domains, the carbohydrate, substrate and ATP binding sites and their 3D structural features according to Gachomo et al. [30].

\section{Scanning electron microscopy (SEM)}

SEM images of upper developing leaves, showing mature trichomes of WT and cvy1 mutant were acquired at different magnifications as previously described [30]. SEM images were taken using a LEO 1450 EP SEM [30].

\section{Cell morphological analysis}

Confocal image analysis was performed on one week after germination of plate grown plants. Pavement-cell shape analysis was performed by staining the samples with $10 \mu \mathrm{M}$ of the lipophilic dye, FM464, for $2 \mathrm{hr}$ in darkness under rocking conditions. The images were acquired using confocal microscopy (inverted Leica SP8 confocal microscope at $488 \mathrm{~nm}, 25 \%$ laser power and emission at $600 \mathrm{~nm}$ ). The F-actin localization was done according to Kotchoni et al. [2]. Images were collected using an inverted Leica SP8 confocal microscope with water-immersion objective. The images were processed and analyzed using ImageJ software.

\section{Determination of flowering time}

Flowering time was assessed by counting the number of rosette leaves when flower bolts were $1 \mathrm{~cm}$ in length or when floral buds were visible at the center of the rosette as previously reported $[30,45]$.

\section{Statistical analysis}

Experiments were performed at least three times. Data were expressed as mean values $\pm \mathrm{SE}$. P values were determined by Student's $t$ test analysis.

\section{Additional files}

Additional file 1: Figure S1. Expression of $C V Y 1$ in various tissues. qRT-PCR analysis of CVY1 gene $( \pm S E)$ of three replicate samples per indicated tissues are depicted. A.U. = Relative expression CURVY1 using internal Actin control in Arbitrary Unit.

Additional file 2: Figure S2. CVY1 regulates cell morphogenesis through actin cytoskeleton bundles. Actin filament bundles were analyzed with ImageJ after the image was thresholded to obtain filament bundles instead of monomeric actin subunit in the pavement cell. Actin bundles were quantified per grid (each grid measuring $25 \mu \mathrm{sp}$ ) as depicted in WT Col-0 (A) compared to cryl (B) pavement cells. Actin bundles crossing the grid boundary were counted for both adjacent grids.

\section{Competing interests}

The authors declare that they have no competing interest.

\section{Authors' contributions}

SOK conceived the study. SOK, EWG wrote the paper. SOK, EWG, L, TK performed the study. SOK, EWG, WMS, analyzed, discussed and assessed the data. EWG, SOK, LJ, WMS contributed reagents/materials/analysis tools. All authors read and approved the final manuscript.

\section{Acknowledgements}

We acknowledged the NSF DBI-0216233 MRI grant "Acquisition of a Scanning Electron Microscope for Collaborative Use at Rutgers, Camden" for the acquisition of the Arabidopsis SEM images in this work. This work was supported by NSF-REU DBI \# 1263163 grant and Rutgers-University start-up funds to SOK.

Received: 14 April 2014 Accepted: 5 August 2014

Published: 27 August 2014

\section{References}

1. Geitmann A: Mechanical modeling and structural analysis of the primary plant cell wall. Curr Opi Plant Biol 2010, 13:693-699.

2. Kotchoni SO, Zakharova T, Mallery EL, El-Din El-Assal S, Le J, Szymanski DB: The association of the Arabidopsis actin-related protein (ARP) 2/3 complex with cell membranes is linked to its assembly status, but not to its activation. Plant Physiol 2009, 151:2095-2109.

3. Zhang C, Kotchoni SO, Samuels L, Szymanski DB: SPIKE1 signals originate from and assemble specialized domains of the endoplasmic reticulum. Curr Biol 2010, 20:2144-2149.

4. Hulskamp M, Misera S, Jurgens G: Genetic dissection of trichome cell development in Arabidopsis. Cell 1994, 76:555-566. 
5. Uhrig JF, Mutondo M, Zimmermann I, Deeks MJ, Machesky LM, Thomas P, Uhrig S, Rambke C, Hussey PJ, Hulskamp M: The role of Arabidopsis SCAR genes in ARP2-ARP3-dependent cell morphogenesis. Development 2007 134:967-977.

6. Zhang C, Mallery E, Reagan S, Boyko VP, Kotchoni SO, Szymanski DB: The endoplasmic reticulum is a reservoir for WAVE/SCAR regulatory complex signaling in the Arabidopsis leaf. Plant Physiol 2013, 162:689-706.

7. Mathur J: The ARP2/3 complex: giving plant cells a leading edge. Bioessays 2005, 27:377-387.

8. Smith LG, Oppenheimer DG: Spatial control of cell expansion by the plant cytoskeleton. Annu Rev Cell Dev Biol 2005, 21:271-295.

9. Szymanski DB: Breaking the WAVE complex: the point of Arabidopsis trichomes. Curr Opin Plant Biol 2005, 8:103-112.

10. Hussey PJ, Ketelaar T, Deeks MJ: Control of the actin cytoskeleton in plant cell growth. Annu Rev Plant Biol 2006, 57:109-125.

11. Basu D, El-Assal Sel D, Le J, Mallery EL, Szymanski DB: Interchangeable functions of Arabidopsis PIROGI and the human WAVE complex subunit SRA1 during leaf epidermal development. Development 2004, 131:4345-4355.

12. Brembu $T$, Winge $P$, Seem $M$, Bones AM: NAPP and PIRP encode subunits of a putative wave regulatory protein complex involved in plant cell morphogenesis. Plant Cell 2004, 16:2335-2349.

13. Deeks MJ, Kaloriti D, Davies B, Malho R, Hussey PJ: Arabidopsis NAP1 is essential for Arp2/3-dependent trichome morphogenesis. Curr Biol 2004 14:1410-1414

14. Frank M, Egile C, Dyachok J, Djakovic S, Nolasco M, Li R, Smith LG: Activation of Arp2/3 complex-dependent actin polymerization by plant proteins distantly related to Scar/WAVE. Proc Natl Acad Sci USA 2004, 101:16379-16384

15. Saedler R, Zimmermann I, Mutondo M, Hulskamp M: The Arabidopsis KLUNKER gene controls cell shape changes and encodes the AtSRA1 homolog. Plant Mol Biol 2004, 56:775-782

16. Zimmermann I, Saedler R, Mutondo M, Hulskamp M: The Arabidopsis GNARLED gene encodes the NAP125 homolog and controls several actin-based cell shape changes. Mol Genet Genomics 2004, 272:290-296.

17. Zhang X, Dyachok J, Krishnakumar S, Smith LG, Oppenheimer DG: IRREGULAR TRICHOME BRANCH1 in Arabidopsis encodes a plant homolog of the actin-related protein $2 / 3$ complex activator Scar/WAVE that regulates actin and microtubule organization. Plant Cell 2005 17:2314-2326

18. Le J, Mallery EL, Zhang C, Brankle S, Szymanski DB: Arabidopsis BRICK1/ HSPC300 is an essential WAVE-complex subunit that selectively stabilizes the Arp2/3 activator SCAR2. Curr Biol 2006, 16:895-901.

19. Schwab B, Folkers U, Ilgenfritz H, Hulskamp M: Trichome morphogenesis in Arabidopsis. Philos Trans R Soc Lond B Biol Sci 2000, 355:879-883.

20. Zhang C, Mallery EL, Schlueter J, Huang S, Fan Y, Brankle S, Staiger CJ, Szymanski DB: Arabidopsis SCARs function interchangeably to meet actin-related protein $2 / 3$ activation thresholds during morphogenesis. Plant Cell 2008, 20:995-1011.

21. Szymanski DB: Plant cells taking shape: new insights into cytoplasmic control. Curr Opin Plant Biol 2009, 12:735-744.

22. Benschop JJ, Mohammed S, O'Flaherty M, Heck AJR, Slijper M, Menke FLH: Quantitative Phosphoproteomics of Early Elicitor Signaling in Arabidopsis. Mol Cell Proteomics 2007, 6:1198-1214.

23. Gomez-Gomez L, Felix G, Boller T: A single locus determines sensitivity to bacterial flagellin in Arabidopsis thaliana. Plant J 1999, 18:277-284.

24. Nuhse TS, Peck SC, Hirt H, Boller T: Microbial elicitors induce activation and dual phosphorylation of the Arabidopsis thaliana MAPK 6. J Biol Chem 2000, 275:7521-7526.

25. Asai T, Tena G, Plotnikova J, Willmann MR, Chiu WL, Gomez-Gomez L, Boller T, Ausubel FM, Sheen J: MAP kinase signalling cascade in Arabidopsis innate immunity. Nature 2002, 415:977-983.

26. Kotchoni SO, Gachomo EW: The reactive oxygen species network pathways: an essential prerequisite for perception of pathogen attack and disease resistance in plants. J Biosci 2006, 31:389-404

27. Schwab B, Mathur J, Saedler R, Schwarz H, Frey B, Scheidegger C, Hulskamp $M$ : Regulation of cell expansion by the DISTORTED genes in Arabidopsis thaliana: actin controls the spatial organization of microtubules. $\mathrm{Mol}$ Genet Genomics 2003, 269:350-360.

28. Zimmermann P, Hirsch-Hoffmann M, Hennig L, Gruissem W: GENEVESTIGATOR: Arabidopsis microarray database and analysis toolbox. Plant Physiol 2004 136:2621-2632.
29. Clough SJ, Bent AF: Floral dip: a simplified method for Agrobacteriummediated transformation of Arabidopsis thaliana. Plant J 1998, 16:735-743

30. Gachomo EW, Jimenez-Lopez JC, Jno Baptiste L, Kotchoni SO: GIGANTUS (GTS1), a member of Transducin/WD40 protein superfamily, controls seed germination, growth and biomass accumulation through ribosome-biogenesis protein interactions in Arabidopsis thaliana. BMC Plant Biol 2014, 14:37.

31. Steinwand BJ, Kieber JJ: The role of receptor-like kinases in regulating cell wall function. Plant Physiol 2010, 153:479-484

32. Becraft PW: Receptor kinase signaling in plant development. Annu Rev Cell Dev Biol 2002, 18:163-192.

33. Huck N, Moore JM, Federer M, Grossniklaus U: The Arabidopsis mutant feronia disrupts the female gametophytic control of pollen tube reception. Development 2003, 130:2149-2159.

34. Rotman N, Rozier F, Boavida L, Dumas C, Berger F, Faure JE: Female control of male gamete delivery during fertilization in Arabidopsis thaliana. Curr Biol 2003, 13:432-436.

35. Guo H, Li L, Ye H, Yu X, Algreen A, Yin Y: Three related receptorlike kinases are required for optimal cell elongation in Arabidopsis thaliana. Proc Natl Acad Sci USA 2009, 106:7648-7653.

36. Deslauriers SD, Larsen PB: FERONIA is a key modulator of brassinosteroid and ethylene responsiveness in Arabidopsis hypocotyls. Mol Plant 2010, 3:626-640.

37. Hematy K, Sado PE, Van Tuinen A, Rochange S, Desnos T, Balzergue S, Pelletier S, Renou JP, Hofte $\mathrm{H}$ : A receptor-like kinase mediates the response of Arabidopsis cells to the inhibition of cellulose synthesis. Curr Biol 2007, 17:922-931.

38. Boisson-Dernier A, Roy S, Kritsas K, Grobei MA, Jaciubek M, Schroeder II, Grossniklaus U: Disruption of the pollenexpressed FERONIA homologs ANXUR1 and ANXUR2 triggers pollen tube discharge. Development 2009, 136:3279-3288.

39. Miyazaki S, Murata T, Sakurai-Ozato N, Kubo M, Demura T, Fukuda H, Hasebe M: ANXUR1 and 2, sister genes to FERONIA/SIRENE, are male factors for coordinated fertilization. Curr Biol 2009, 19:1327-1331.

40. Hematy $\mathrm{K}$, Hofte $\mathrm{H}$ : Novel receptor kinases involved in growth regulation. Curr Opi Plant Biol 2008, 11:321-328.

41. Lindner $H$, Muller LM, Boisson-Dernier A, Grossniklaus U: CrRLK1L receptor-like kinases: not just another brick in the wall. Curr Opi Plant Biol 2012, 15:659-669.

42. Shiu SH, Bleecker AB: Receptor-like kinases from Arabidopsis form a monophyletic gene family related to animal receptor kinases. Proc Natl Acad Sci USA 2001, 98:10763-10768.

43. Kotchoni SO, Kuhns C, Ditzer A, Kirch H-H, Bartels D: Over-expression of different aldehyde dehydrogenase genes in Arabidopsis thaliana confers tolerance to abiotic stress and protects plants against lipid peroxidation and oxidative stress. Plant Cell Environ 2006, 29:1033-1048.

44. Baulcombe DC, Saunders GS, Bevan MW, Mayo MA, Harrison BD: Expression of biologically active viral satellite RNA from the nuclear genome of transformed plants. Nature 1986, 321:446-449.

45. Kotchoni SO, Larrimore KE, Mukherjee M, Kempinski CF, Barth C: Alterations in the endogenous ascorbic acid content affect flowering time in Arabidopsis. Plant Physiol 2009, 149:803-815.

doi:10.1186/s12870-014-0221-7

Cite this article as: Gachomo et al:: The Arabidopsis CURVY1 (CVY1) gene encoding a novel receptor-like protein kinase regulates cell morphogenesis, flowering time and seed production. BMC Plant Biology 2014 14:221. 